\title{
Multipath Cluster-based Hybrid MAC Protocol for Wireless Sensor Networks
}

\author{
Ch Rambabu ${ }^{\mathrm{a}}$, V.V.K.D.V.Prasad ${ }^{\mathrm{b}}$, K.Satya Prasad, ${ }^{\mathrm{a}, \mathrm{b}, *}$ \\ ${ }^{a}$ Research Scholar, Department of ECE, JNTU Kakinada, Andhra Pradesh, India \\ ${ }^{b}$ Professor, Department of ECE, Gudlavalleru Engineering College, Gudlavalleru, Vijayawada, Andhra \\ Pradesh, India \\ ${ }_{a, b, *}$ Rector, VFSTR deemed to be University, Guntur, Andhra Pradesh, India
}

Received: 14 October 2019; Accepted: 01 November 2019; Published: 08 February 2020

\begin{abstract}
The WSN (Wireless Sensor Network) is the most appearing expertise that has potential applications broad ranges that include environment examining, smart spaces, medical systems, and robotic study. The efficient energy is a consideration of vital design for WSN. In WSNs, the collision is occurred due to data transmission from the sensor nodes and the traffic at SINK node is high due to the transmission of excess data by the sensor nodes. An important division of the consumption of resources in a WSN is managed by the mechanism of MAC (Medium Access Control). An existing MAC protocols initiated for the utilization of WSNs single channel for the transmission of data. This is basically because of the reality that efficient energy is measured to be the issue of essentiality in WSNs. A new multi-channel MAC procedure MPCB-HM is proposed which utilizes CSMA/CA (Carrier Sense Multiple Access/ Collision Avoidance), to exchange the data, the activity of TDMA (Time Division Multiple Access) sequencing nodes and also FDMA (Frequency Division Multiple Access) to allow collision-free exchange simultaneously. The nodes have multiple communication channels, so that the high data traffic can be shared in multiple channels. This reduces the overhead in the nodes and the Energy consumption is minimized by this method and collision free transmission is achieved. With the help of intra-cluster communication and inter-cluster communication, the MAC mode control is responsible for shifting of mode from TDMA to CSMA and vice versa. The Cluster-based topology is implemented which helps in improving the scalability and energy efficiency. By utilizing the simulator of NS2, the process is estimated and the outcomes have shown that the procedure of MAC is improved by overall network presentation compared to the other protocols.
\end{abstract}

Index Terms: Wireless sensor network, Multi-channel, MAC Protocol, TDMA, CSMA/CA, FDMA, Energy efficiency, Collision avoidance, Cluster Head, DEEH-CB, Adv-MMAC, RBDCEER, QTSAC, MPCB-HM.

(C) 2020 Published by MECS Publisher. Selection and/or peer review under responsibility of the Research Association of Modern Education and Computer Science

\footnotetext{
* Corresponding author. Tel.: +91-9490724428
}

E-mail address: chrambabuec41@gmail.com 


\section{Introduction}

In customary applications of WSN, efficient energy has been significant for lengthening the system duration, where the requirements of QoS (Quality of Service) that is bandwidth and postponement in controls are significantly less important. Though, the present WSN multimedia like controlling the vehicular travel flow on the highways to battlefield acumen needs trustworthy and real-time exposure to the events. These purposes generate dimensions heavy data in a diminutive time that would cause a high channel contention degree and possibility of the high collision of package. But other side, various procedures [1] of MAC have created for WSNs along with a purpose to enhance the efficient energy, throughput, and scalability. Thus, a lot of healthcare purposes [2] of WSN produce burst traffic that involves high throughput, low delay, and the rate of high delivery. The WSNs could not be provided a trustworthy and on-time delivery of the data with a solo channel with a high rate of data constraints due to the collisions and restricted bandwidth. Hence, the communication of multi-channel in WSN that was involved the examiners at the time of the last supporting decade of the burst traffic in WSN.

As the WSN application turns into insidious, the density of node (no. of nodes/unit region) would be likely to enlarge. This would offer the latest dispute for the layer of MAC design of the protocol. Although, various conventional protocols of MAC have aimed, and they use only a sole rate of recurrence (channel) and the utilization of the solo channel restricts the presentation. Moreover, many protocols of multi-channel MAC level which was initiated to raise the act of WSN. This has been noticed that protocols of multi-channel MAC are having efficiency in a smaller amount of energy than the protocol of sole channel MAC under all the conditions of traffic. Whereas, whenever the density of the network rises, the protocol of a single channel will not give a substantial efficient outcome as interference occurs. Hence, it is very essential for designing a multi-channel MAC protocol efficiently for attaining both the high performance and efficient energy in dissimilar conditions of the traffic. The communication of multichannel is a competent way to moderate the interference effect and disputation on the wireless medium through planning transmission of the interfering over dissimilar frequency channels for improving the throughput.

A WSN is an association consists of several sensors that have been arranged in an Ad-hoc way. Every sensor is having an ability of equal sensing which would sense the conditions of environment like temperature, pressure, sound, and would gather the information from the fields of dissimilar and get ahead of the information that is collected to the sink node or base station by numerous nodes of the head of cluster. The node of the sensor will have fundamental parts which are named as memory, transmitter, processor, receiver, power supply, and sensing unit. Furthermore, a WSN consists of various practices and algorithms. Also, it can self-organize. It is used chiefly in the functions of the military like forest fire detection, battlefield surveillance, industrial process controlling and the management of agricultural irrigation where there is high risk.

The primary concern of WSN is consuming the power which would affect the duration of WSN. Thus, additional power is crucial to collect the data, aggregation of data and forwarding the data. When a node sensor is in sleep status or would practice nothing, its power is minimized. Hence, the power that is effective responsive of the techniques of routing with the clustering is required for building the less energy fair clusters through taking into consideration of the end-to-end delay function \& function of the rate. Every head of the cluster aggregates the arriving data in intercommunication of the cluster from the members of cluster and it will forward the data aggregation to node of sink by relay nodes where the communication of intracluster has been passed out in the cluster where every node would gather and send the data to their head of cluster. Routing is utilized for transmission of the sensed aggregated data to the sink through finding a prearranged way. The algorithm of DFS (Depth First Search) is been utilized for finding the best way amongst several probable routes amidst the source nodes to purpose because of the network's graph structure. It would reduce the cost of the way and also delay additionally. It also enhances the duration of the network and throughput with no delay. The regulations are also utilized here to find the short way as a decision making of rule-based will be providing more exact outcomes in the design of networking schemes [3] besides. 
A node of sensor is normally created of 3 standard components in WSNs: a subsystem of sensing for the acquirement of data from unimportant ambiance, a subsystem that is been dealing out for the processing of local data and a subsystem of wireless statement for the essential data transmission [4]. A WSN would consist of many sensor nodes along with a very restricted range of transmission that is been enhanced in the area of management and generally that should be having formless infrastructure. And these systems would have heavy utilization in civil or military surveillance, tracking the target, protection of territory/premises, natural disasters forecasting and monitoring the health of humans [5,6]. Specific military instances would contain correlated spatially and troop that is coordinated, movements of tank and the essential information collection about the geographical equipment position. The nodes of the sensor could sense and perceive the environment for estimating the disaster before the occurrence of natural disasters. For example, the sensors' consumption all alongside the volcanic region could identify the earthquakes increase and eruptions. Specific sensors could be helping control a patient's health by monitoring human health [6].

Every node of sensor reports took place phenomena and also performs the process of local that includes fusion of data and quantum in WSNs. The data aggregation is been sent directly to (BS) Base Station or using another relay node. At last, all pooled facts which have been connected along with a constraint would get practiced and the outcome worth is fairly approximated accurate. The node failure has about nil impact on the approximate value, whereas it would cause the area of coverage loss and might increase the delay in diagnosing event.

The aggregation of data in WSNs would involve uniting the facts that fit into a special event. The purpose of primary aggregation is to lengthen the lifetime of the network by minimizing the resource expenditure (for example battery energy or bandwidth) and quantity of diffusion packages. The aggregation of data practices the result on the excellence of service factors in spite of such as data correctness, latency, tolerance of fault and security [7].

Because of the broad spread of WSNs utilization \& sensor's question of parameter, the obligation comes up for latest and efficient energy procedures around the aggregation of data being subjective. Hence, utilizing programs of the power-aware and algorithms is momentous enormously [8, 9].

One of the familiar methods to attain the aggregation of data is that sensor nodes will be divided into various groups \& in every group, one node will be chosen as ahead of the cluster for the aggregation of data tasks. $\mathrm{CH}$ (Cluster Head) role would turn in the region of the nodes to make sure the division of the load amidst the nodes. In latest times, algorithms of clustering have been a focus for the attention of several examiners. They have proved that the clustering is an effectual method in the increase in the scalability, balancing the load amongst the nodes and the time prolonging of WSNs $[10,11]$. The quantity of the methods would suppose that a node could transmit its data directly to the BS, whereas it is not powered capable because transmission of longdistance data. To resolve these challenges, few methods would let the nodes to relay their data in the multiple hops to the BS [12]. As these approaches would require re-transmitting the packets of control to route the construction functions some power wasting still available in this phase.

One more primary concern of WSNs is the crisis of energy and emissions of carbon that have become universally typical concerns $[13,14]$. The millions of sensors are arranged to manage the tiny grid, and the infrastructure of the statement is an important component to implement the energy internet $[15,16,17,18,19]$. The triumphant elegant energy management realization would rely on omnipresent and statement of trustworthy amongst the millions of sensors that have been deployed in the field [14]. The tools that have pervasive sensing could be connected and controlled all kinds of devices that are smart grid utilizing anywhereanytime-to-anything style of connectivity [20, 21. 22. 23, 24, 25]. Furthermore, due to the sheer number of individuals, a network of huge data $[26,27]$ has been figured, which would combine cloud computing [23, 28] along with Internet of Things (IoT) $[29,30] \&$ would bring immense ease for the management of elegant power. Whereas, the big amount of facts and incredible amount of statement would be consuming a vast deal of power $[31,32,33]$. Hence, the Internet of power would also rely on millions of actuators and sensors, which been are arranged in the field, \& for proficient message $[34,35,36]$. 
One of the significant elements of an elegant infrastructure is the nodes of the sensor of the wireless, which also have an easy structure, of lesser price and also proper for a broad range of operation settings. Hence, they would finish the smart grid monitoring realization [37, 38, 39]. WSN (Wireless Sensor Networks) are frequently utilized for smart grids, monitoring the environment, operational surveillance, home and industrial automation [40, 41, 42], and also community systems, like networks of community mobile [43, 44, 45]. These associations are generally collected from little sensors that have restricted sources in terms of power procedures, storage of the data, and transmission of radio [46, 47]. The strict restraint in WSNs is the energy which is limited $[48,49,50]$. It is unfeasible and impractical for most applications to reload them with energy. It shows that it is highly difficult for designing the system of energy-efficient submission with a lifetime [50, 51]. Substantial study on the saving of energy has been performed, chiefly on the optimization of routing algorithm in WSNs [52], fusion of data [53], optimization of the MAC [32], \& the cross-layer methods of the optimization that would unite the numerous stages [54]. The optimization of MAC is one of the important processes. Recurrently utilized MAC method of optimization is to make use of nodes of sensor to control the functioning (i.e., communication \& sensing) elements on \& off like clockwork [54, 55, 56, 57, 58]. When it is contrasted with the state of working (active), the functioning units of power utilization whenever they are turned off (sleep) is lesser by other than one magnitude command. The nodes would set to the sleep state as much as probable to save the energy.

In this section, Collision, Traffic and the Energy consumption are the major objectives in designing this paper. When multiple data is transmitted to the SINK node by the sensor nodes instantly, then there is a chance of occurrence of Collision. The traffic is higher near the SINK node because multiple data packets from various sensor nodes is transmitted to the SINK node via different multiple channels. The energy is consumed rapidly by transmitting and receiving large amounts of data and by exchanging multiple data using a single channel. By focusing on the above objectives, this proposed method is implemented.

\section{Literature Survey}

We planned an efficient energy algorithm of the cluster-based hierarchical routing for WSNs in a distributed complete manner in this paper. The primary challenge of a protocol scheme is at the time of routing tree construction. CH's will be selected at the tree boundaries that are based on useful local information. The procedure of decision-making for $\mathrm{CH}$, we would be utilizing the multiple criteria, like energy of every node's residual and its space to the BS with the tree creation of routing. Every node would be deciding without help whether to turn out to be a $\mathrm{CH}$.

Regular nodes combine to the suitable CHs. Because of the routing combination and the scheme of clustering necessity, the numerous packets of the control decrease and extra power will be kept in every node of the sensor consequently.

We recommend an advertisement of energy-efficient based multi-channel MAC (Adv-MMAC) procedure to powerfully utilize the multiple paths for WSNs to enhance the efficient energy, latency, and throughput. The WSN radios frequency that is operating could be altered over dissimilar channels so the communication could be made delivery more records to the node of sink in short intervals simultaneously.

In [59], the author proposed with an extended edition of the previous effort an advertisement based procedure of the multi-channel MAC for WSNs (Adv-MMAC). We would be analyzing the best energy capable Adv-MMAC presentation thoroughly.

In [60], the author proposed the primary propose of our Adv-MMAC practice will be based on the announcement based MAC (ADV-MAC) procedure. The Adv-MMAC utilizes a method of conflict to choose the modes to correspond the broadcasts on dissimilar channels. The node also has the information for transmission of first changes its interface and challenges the announcement channel to convey the x packet of ADV. 
In $[61,62]$, the author proposed the ADV and packages of data have been broadcasted at the time of a time frame in a parallel way. We estimate the act of the planned Adv-MMAC procedure with the simulation extension in NS-2.34 and give a research of comparison along with the projected multi-channel recently procedure of MAC, Queen MAC and a solo channel announcement based division of time MAC (ATMA) practice that has been intended over ADV-MAC.

The latest DCEMRA (Delay Constrained Energy-efficient Multi-hop Routing Algorithm) has been planned to perform a valuable routing in WSNs. A recommended procedure commences a latest procedure known as a stoppage forced trustworthy routing algorithm which decreases the consumption of energy through building efficient clusters lacking growing the end-to-end delay.

The latest computational methods are established for this purpose in this form to find the spaces among the cluster heads \& the elements of cluster, one cluster head to the other cluster head and the cluster heads to the sink node. Furthermore, the finest undeviating way is been found in this task by locating all the probable short ways and affecting the regulations for finding the shortest best way by utilizing a decreased function.

The regulations are also utilized in addition to this task to make a resolution that is suitable for finding the greatest and optimal way from the set of all short ways through taking into consideration energy, metrics and the distance of delay. The primary benefit of the recommended routing algorithms includes the raise inconsistency, ratio of packet delivery, lifetime of network and energy consumption reduction and delay.

A novel QTSAC (Quorum Time Slot Adaptive Condensing) based intermediate contact organize procedure has been recommended to achieve the minimization of delay and efficiency in the energy for WSNs. The primary assistance here is as follows:

(1) The QTSAC procedure concentrates the QTS to the span where the transmission of data nodes, which would increase the connection, slots, for enhancing system act.

The QTS is been distributed randomly over the entire cycle in existing Quorum Systems. The significant originality of the QTSAC-based MAC practice (QTSAC procedure for tiny) is the allotment of QTS as per the nodes of the procedure of data span, then consistent allocation over the whole cycle. Whenever the data assembling happens in WSNs, the data is being sent out from the regions that are distant from the sink to the sink. Hence, nodes' functions of the data in the regions which were close to the sink are contemplated in the second half of the series, as the nodes' data functions of in regions that are distant from the sink are contemplated in the first half of the series. Hence, the creation of QTS in the QTSAC procedure is correlated to the regions of the nodes. It implies that the nodes' QTSs in the regions which are distant from the sink are determined in the first half of the cycle, where the QTSs of nodes in regions that are close to the sink are concerted in the second half of the cycle. More QTSs are owed through the time the nodes data function, as a smaller amount or no QTSs are assigned through the stage with no operation of data. This adaptive QTSs allocation could conserve power and lengthen the lifetime of the network.

(2) The procedure of QTSAC creates full spending of the nodes' outstanding power in the marginal region of the system and would increase the QTS's in numbers, thus decreasing the latency of network with no effect on the lifetime of network.

The QTS's numbers in an earlier study of the nodes in an association are alike or accordingly allotted to every node's workload. The procedure of QTSAC would not reduce the QTSs number of nodes in regions that are distant from the sink; in its place, it would increase the number of QTSs. Hence, it will not only make the utilization of the outstanding power yet also minimizes the latency of network for improving the act of the procedure.

(3) It is verified as the holdup of network and efficiency of energy could simultaneously improve by our extensive theoretical analysis and the study of simulation. When it is compared with the earlier protocol of the Quorum-based MAC, the postponement could reduce with as much as $23 \%$ and $10 \%$. It develops an act in 
terms of the above- declared elements with no harm to the lifetime of network, which was tough to attain in earlier researches.

\section{Proposed Algorithm}

We have addressed the rates of big data test and burst traffic in multi-hop WSNs here in this work where a huge burst packets' requirements to be moved to the ultimate objective. We would be introducing a sink of the multi-interface for additional development of the arrangement throughput whenever all the traffic is designed to the sink. The response of the sink to optimize, we have customized the portion of channel scheme to remain the sink interfaces in the mode of reception and minimum one neighbor of every edge in the mode of the transmission. Hence, the plan of channel allocation would allow the sink to accept the data frames constantly. The propagation of beacon and discovery of neighbor phase in network creation the first node to be triggered in the network that is sinking \& is on the depth O. The sink screens a beacon which circulates in a multi-hop approach for reaching all the network nodes. Along with the messages of elongated control to swap the information of the neighborhood to avoid network overloading, we utilize the bitmaps to signify the neighbors. By utilizing the order of local propagation (which is a listing of all the node addresses of the system), each node can construct \& control a bitmap that would represent all the network nodes. Every bitmap index would correspond to the address of the node with the same index in the order of propagation. Whenever a node would be receiving a beacon from the other node, it would consider that this node is a neighbor. Every node would comprise the bitmap of its I-hop neighbors in the beacon to construct 2-hop neighbor's list. Thus, whenever a node collects all the beacons from its neighbors, then it will have the ability to assemble the 2-hop neighbors list. Every node has to contain the bitmap of its I-hop and 2-hop neighbor's bitmaps in the beacon it launches to construct 3-hop neighbor lists. To make 1-hop, 2-hop, and 3-hop neighborhood interference by utilizing the beacon and the order of propagation, we keep away from utilizing additional control traffic like messages of HELLO which would be traditionally by the protocols of routing. Neighboring information has been efficiently exchanged among the nodes with no collision and with light overhead by using codification of the bitmap and throwing beacons in a TDMA mode. All the nodes wanted to correspond along with the similar node participate in the identical channel to contact the medium and share the information by utilizing the algorithm of CSMA/CA. Topology has been systematized into various depths that are based on the topology of Zig Bee Custer-Tree. The NC must stay in the reception method to raise the throughput and the nodes in-depth 1 has to alternate among the manner of sending and the approach of reception to remain as a part of them in the broadcast form. Hence, we have separated the system into 2 groups, Group1 would include the nodes of odd deepness that were descendants of an even child of an interface (B, D, F are even children of an interface), and that will include the nodes of an even depth that the descendants of an interface odd child. All the other nodes have been comprised in Group2. Every node has a 3-hop neighborhood bitmap for channel allocation which would enable it to select its channel with dynamism. The highest priority node in the 3-hop neighborhood that first selects its channel. Priorities would be assigned based on the addresses of the association. The node with the address of the minimum system is having the uppermost concern. A node will proceed to its allocation of the channel when it would become the node with the address that is smaller amongst 3 hop neighborhood that is not yet allocated a channel. A bitmap which is representing all the nodes is utilized for announcing the nodes have finished the process of channel allotment for every node to recognize whether it is its turn to select a channel. Whenever a node selects its channel, it will broadcast it in the beacon frame. 


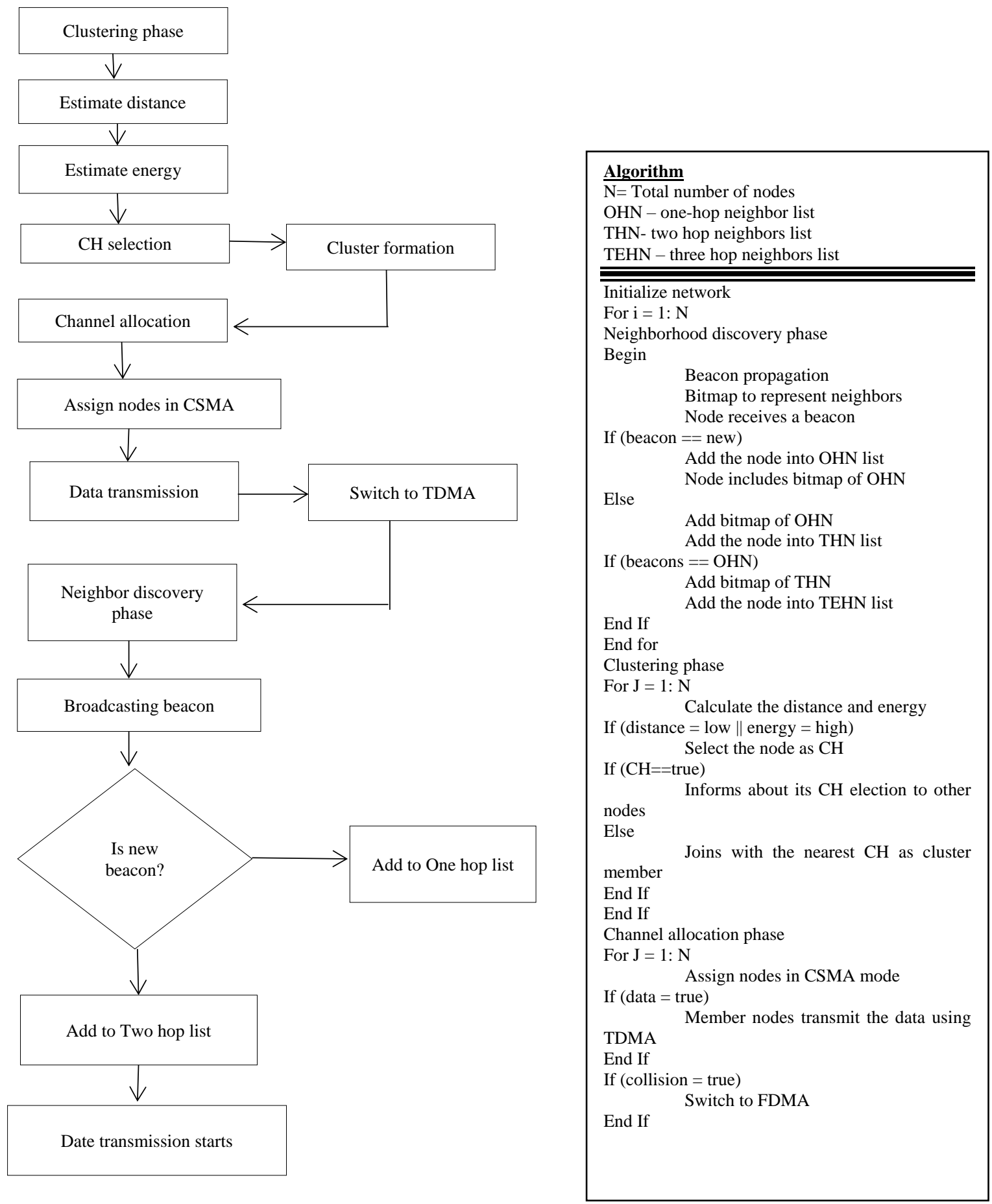

Fig.1. Work flow of proposed method 


\section{Intra cluster communication:}

After the establishment of the route towards the sink, the data is sent to the Cluster Head $(\mathrm{CH})$ by the nodes within the cluster and later it is forwarded to the sink by hop by hop. The intra cluster communication is coordinated by the $\mathrm{CH}$ and is based on TDMA. The following paragraphs describe the protocol.

\section{TDMA slots reservation step:}

The request phase is announced by the $\mathrm{CH}$ and it is operated in CSMA mode upon the CC channel. With the help of learned route, the data is transmitted to the sink through the inter cluster communication if cluster member has an effective route. Else the data is transmitted through the $\mathrm{CH}$ by the node. The $\mathrm{CH}$ receives a REQUEST sent by the node. The sum of requested time slots, the QoS level of the data (real-time, non-realtime) and the route towards the sink if present is announced in this situation.

\section{TDMA slots allocation and transmission step:}

Depending upon the expected availability time of every cluster, the TDMA time slots is allocated and the transmission channels are scheduled for every single node when REQUEST messages are received. The $\mathrm{T}$ _SCHED message is broadcasted by the $\mathrm{CH}$ and informs the cluster members regarding the TDMA time slots allocation and channels scheduling. Once the message is received, the cluster nodes and the $\mathrm{CH}$ are synchronized and the data transmission is initiated in the particular time slots through the designated channel. It considers that initially all clusters are using a CSMA mode. The MAC mode control is responsible for shifting of mode from TDMA to CSMA and vice versa. If the amount of collision inside the cluster goes beyond the collision threshold value, then the cluster will shift the node from CSMA to TDMA.

\section{Inter cluster communication:}

The Inter cluster communication is essential for reaching the sink. Therefore, there is a need for synchronizing the channel in-between the node which is outside of the layer on the upstream cluster as well as the access node on downstream cluster. There is a possibility of two inter cluster communication. In the initial way, the data is essential for the node for the transmission and moreover it requires an effective route.

The realization of the TDMA mode is performed in this procedure when the data is transmitted between the node and the $\mathrm{CH}$. The node which is exterior to the TDMA slots operates in CSMA mode and synchronized to CC channel.

\section{FDMA}

FDM is a technique of the physical layer where FDMA is an access system in the data link layer. FDM merges the loads from unusual channels of low bandwidth and will be transmitted by utilizing a channel of high bandwidth. Here, collision free exchange of data is achieved by using FDMA.

\section{Results and Discussion}

This work has been put into practice by utilizing the Network Simulator- 2 with the support of simulation patch files. NS-2 is been utilized for experimental confirmation. It is an open platform of system simulation that would afford an open-source, and functioning at package level. This would present the users with command that could be executed by ns that takes the input case and its name as Tcl scripting language. The users are forwarding the name of the Tcl script as an input case of ns2 command that can be executed using ns. In many other cases, the simulation trace file can be generated. 
The ns 2 outcomes are text based or animation based outcomes after the process of simulation. The tools like Nam and X-graphs must be utilized for interpreting these results interactively or graphically. The users could extract a relevant subset of text based data and alter to possible presentation for analyzing a specific behavior of network.

Table1 illustrates that the system constraints utilized in our simulations. The Application Traffic as is used as CBR (Constant Bit Rate) that could provide the support to manage the traffic in network, Routing Protocol as AOMDV and it is utilized to route the level in system, Routing methods are QTSAC, ADV-MMAC, RBDCEER, DEEH-CH, and MPCB-HM in our simulation, and this routing schemes are professionally been used to perform the network conclusions. Each packet size of 1024 bytes is considered for transmission with a channel data rate of $30 \mathrm{Mbps}$. Here, the time taken for transmitting one packet is $0.1 \mathrm{~ms}$. In this simulation, the overall network processing time is 20 secs. The speed of the network is indicated using the step time. The $35 \mathrm{~m} / \mathrm{s}$ of network speed is analyzed by considering the packet size, transmission time, overall simulation time. The average distance between the nodes is $250 \mathrm{~m}$.

In a network scenario, the initial process is showing the operation of sensor nodes in the $500 \mathrm{~m} \times 500 \mathrm{~m}$ in the network along with 21 nodes.

Table 1. Simulation table of Proposed Network

\begin{tabular}{|l|l|}
\hline PARAMETER & VALUE \\
\hline Application traffic & CBR \\
\hline Transmission rate & 1024 bytes/0.1msec \\
\hline Radio range & $250 \mathrm{~m}$ \\
\hline Packet size & 1024 bytes \\
\hline Channel data rate & $30 \mathrm{Mbps}$ \\
\hline Maximum speed & $35 \mathrm{~m} / \mathrm{s}$ \\
\hline Mac protocol & $\begin{array}{c}\text { Mac/802_11, Mac/Csma/ca, } \\
\text { Mac/Tdma, Mac/Fdma }\end{array}$ \\
\hline Routing protocol & AOMDV \\
\hline Simulation time & $20 \mathrm{secs}$ \\
\hline Number of nodes & 21 \\
\hline Area & $500 x 500$ \\
\hline
\end{tabular}

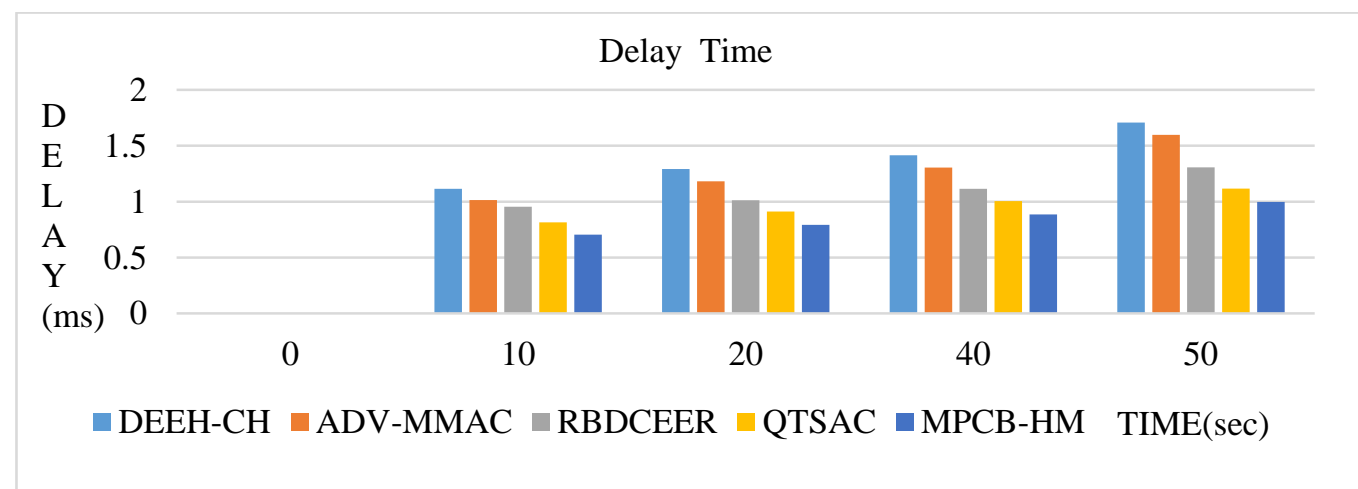

Fig.2. Delay Performance 
In Figure 2, the graph explains and signifies end 2 end delays and it would show the time of simulation versus delay. The presentation of the projected algorithm advances the time of delay that means diminish the delay among the communications nodes compare to algorithms of existing. With the help of CSMA or CA, TDMA modes, the multi-channel allocation is performed and the delay is reduced by $30 \%$ in the proposed method compared to the existing methods.

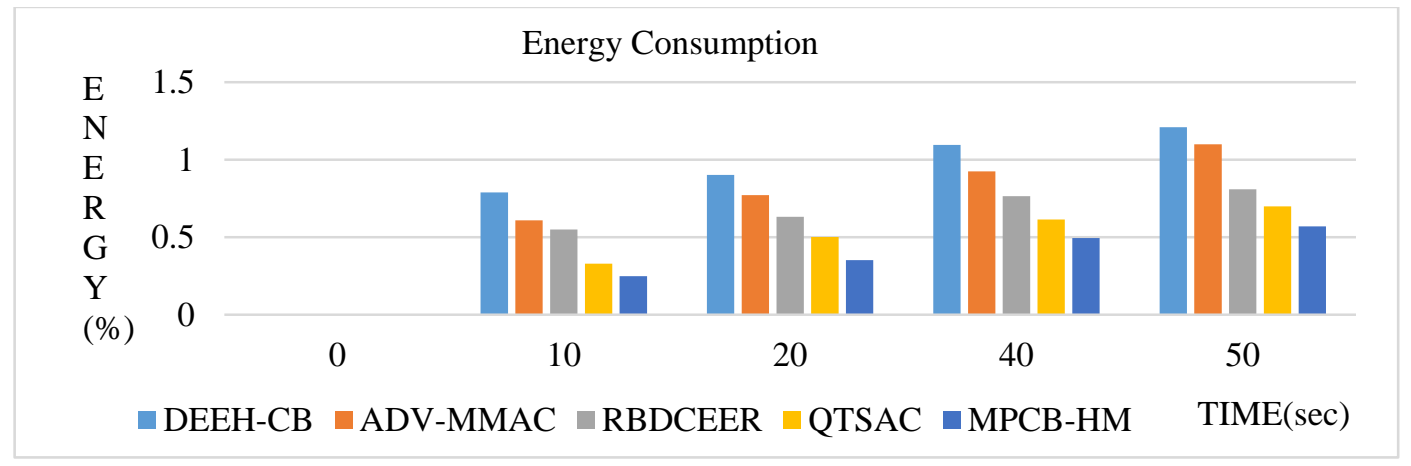

Fig.3. Energy Consumption

Figure 3 would be showing and representing the utilization power and it would show the time of simulation versus the ratio of energy level. The proposed algorithm performance will improve the ratio of loss which means lessen the power spending compare to presented algorithms. The energy consumption is reduced in the proposed method due to the collision free transmission of the data from $\mathrm{CH}$ nodes to the SINK node and the energy levels are increased by $35 \%$.

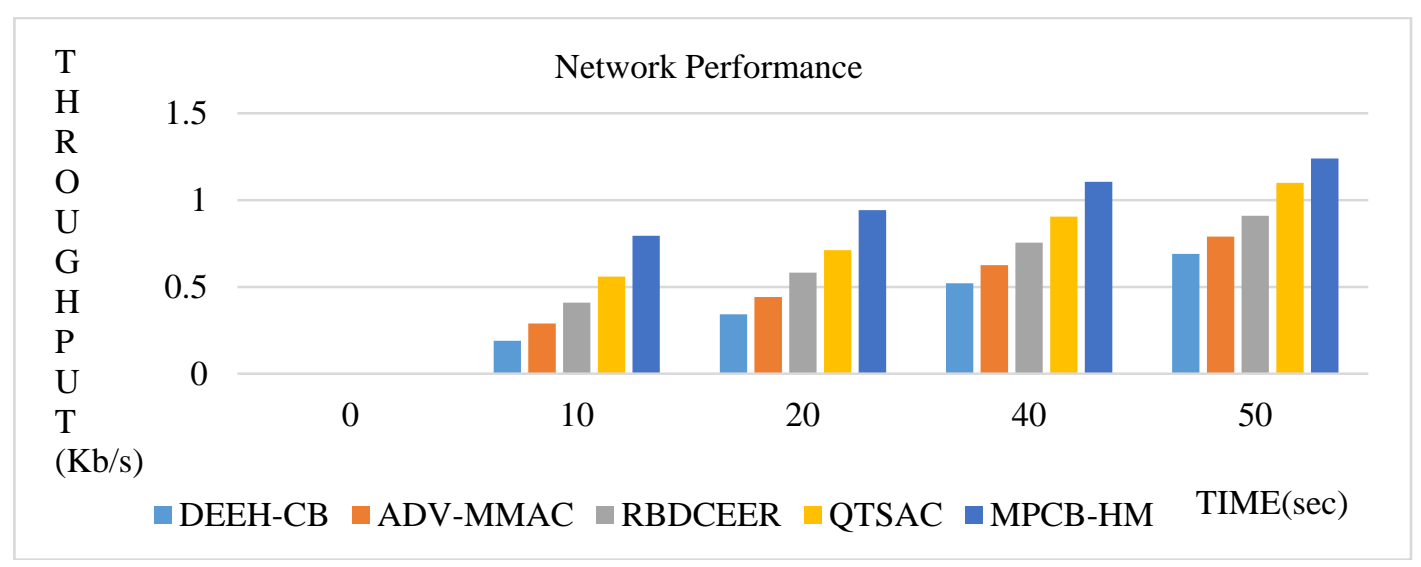

Fig.4. Network Performance

Figure 4 proves and characterizes the performance of the network and that confirms the time of simulation versus throughput. The act of planned algorithm will be improving the performance of the network and it is meant that saving the rate of data within the given time interval compares to the existing algorithms. A throughput with an average of $40 \mathrm{~Kb} / \mathrm{s}$ is possessed by this method. The data is transmitted without any loss and the performance of the network is efficient compared to the existing methods. 


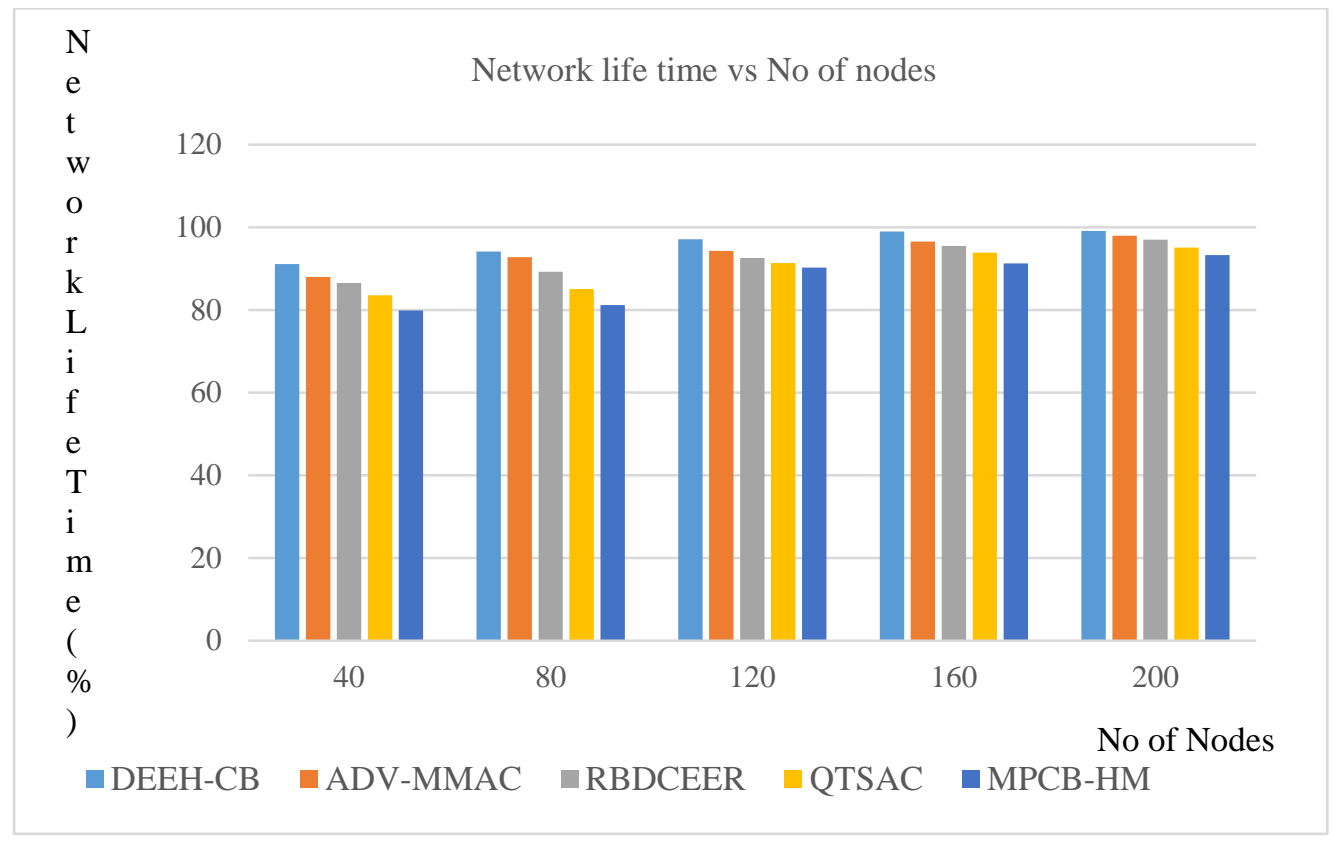

Fig.5. Network lifetime vs Number of nodes

Figure 5 proves and characterizes the performance of network life time vs number of nodes. The act of planned algorithm will be improving the performance of network life time and it is meant that saving the life time when it compares to the existing algorithms. Reduced energy consumption and higher load balancing among the SNs is the main objective for increasing the lifetime of the network. The network lifetime is enhanced by $20 \%$ due to the variations in number of transmitting and receiving nodes and the data flow varies with the increase in the number of nodes.

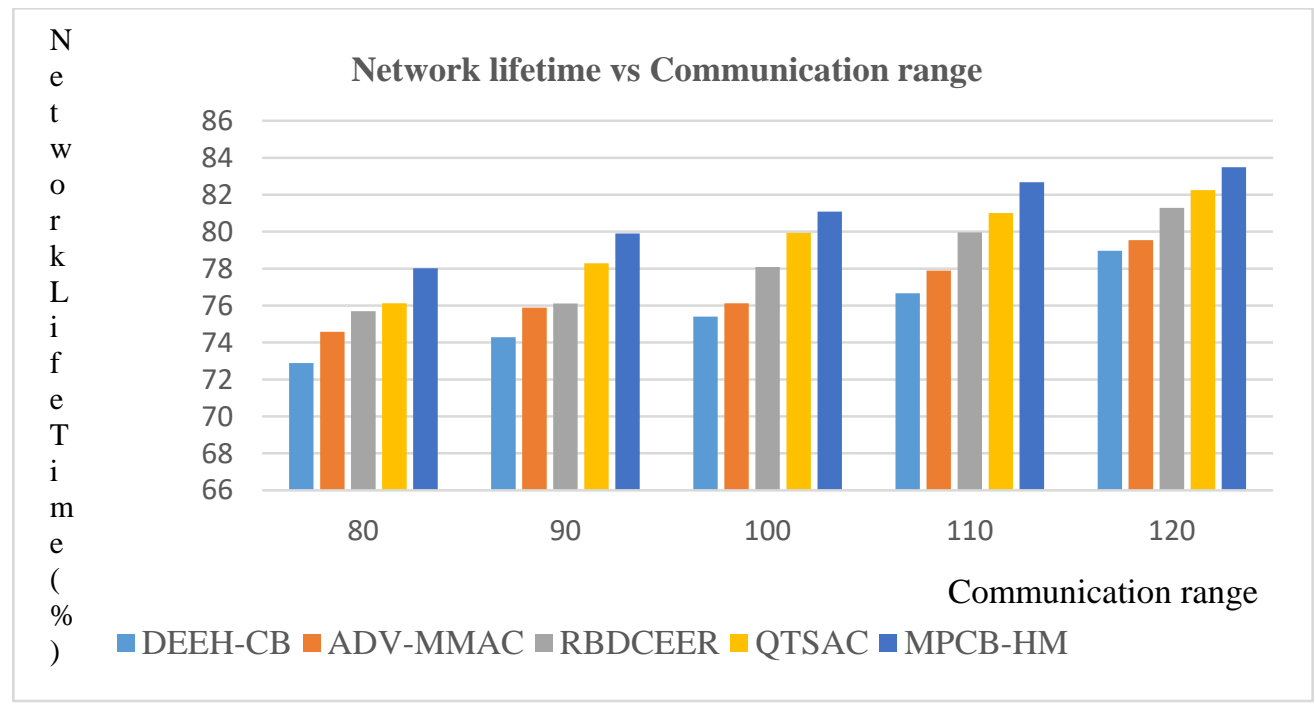

Fig.6. Network lifetime vs Communication range 
Figure 6 proves and characterizes the performance of network life time vs communication range. The act of planned algorithm will be improving the performance of network life time as well as the communication range and when it compares to the existing algorithms. With the increase in the overall communication range, the network lifetime is increased by $30 \%$. Even though the distance between the nodes is increased, the lifetime of the network is increased in the proposed method compared to other methods.

\section{Conclusion}

The MPCB-HM performance and a protocol of hybrid multi-channel MAC for WSNs is assessed in this paper. This research work proves that the proposed MPCB-HM protocol considerably giving better performance than the other previously proposed protocols and also helps the network to control the occurrence of collision and ensure the smooth data flow. Clustering technique used in this protocol divide the network into multiple groups for ease management of the network. The use MAC protocols ensure hassle-free data transmission between Inter and Intra clustering communication. This protocol is tested under various dynamic network conditions and under different network load to check the stability of the protocol. The results have been shown that MPCB-HM noticeably minimizes the consumption of the energy when it is compared to the other procedures.

In our future works, we plan on evaluating the MPCB-HM protocol under burst traffic to evaluate the stability of this protocol and planned to improve the energy efficiency.

\section{References}

[1] Demirkol, I., Ersoy, C., \& Alagoz, F. (2006). Mac protocols for wireless sensor networks: A survey.IEEE Communications Magazine, 44(4), 115-121.

[2] Hanjagi, A., Srihari, P., \& Rayamane, A. S. (2007). A public health care information system using GIS and GPS: A case study of Shiggaon. In P. C. Lai \& S. H. Mak (Eds.), GIS for health and the environment. New York: Springer.

[3] Anand, K., Ganapathy, S., Kulothungan, K., Yogesh, P., Kannan, A.: A rule based approach for attribute selection and intrusion detection in wireless sensor networks. Procedia Eng. 38, 1658- 1664 (2012).

[4] Anastasi G, Conti M, Di Francesco M, Passarella A. Energy conservation in wire-less sensor networks: a survey. Ad Hoc Netw 2009;7:537-68

[5] Schaffer P, Farkas K, Horváth Á, Holczer T, Buttyán L. Secure and reli-able clustering in wireless sensor networks: a critical survey. Comput Netw2012;56:2726-41.

[6] Yick J, Mukherjee B, Ghosal D. Wireless sensor network survey. Comput Netw2008;52:2292-330.

[7] Ozdemir S, Xiao Y. Secure data aggregation in wireless sensor networks: acomprehensive overview. Comput Netw 2009;53:2022-37.

[8] Albath J, Thakur M, Madria S. Energy constraint clustering algorithms for wire-less sensor networks. Ad Hoc Netw 2013;11:2512-25.

[9] Khediri SE, Nasri N, Wei A, Kachouri A. A new approach for clustering in wirelesssensors networks based on LEACH. Proc Comput Sci 2014;32:1180-5.

[10] Younis O, Fahmy S. HEED: a hybrid, energy-efficient, distributed clus-tering approach for ad hoc sensor networks. IEEE Trans Mobile Comput2004;3:366-79.

[11] Yu J, Qi Y, Wang G, Gu X. A cluster-based routing protocol for wireless sensornetworks with nonuniform node distribution. AEU - Int J Electron Commun2012;66:54-61.

[12] Gu X, Yu J, Yu D, Wang G, Lv Y. ECDC: an energy and coverage-aware dis-tributed clustering protocol for wireless sensor networks. Comput Electr Eng2014;40:384-98.

[13] H. Dai, G. Chen, C. Wang, et al. "Quality of energy provisioning for wireless power transfer." IEEE Transactions on Parallel and Distributed Systems., vol. 26, no. 2, pp. 527-537, 2015. 
[14] Z. Zhou, J. Gong, Y. He, Y. Zhang. "Software defined machine-tomachine communication for smart energy management." IEEE Communications Magazine., vol. 55, no. 10, pp. 52-60, 2017.

[15] S. He, X. Li, J. Chen, et al. "EMD: energy-efficient P2P message dissemination in delay-tolerant wireless sensor and actor networks." IEEE Journal on Selected Area in Communications, vol. 31, no. 9, pp. 75-84, 2013.

[16] J. Gui and K. Zhou, "Flexible Adjustments Between Energy and Capacity for Topology Control in Heterogeneous Wireless Multi-Hop Networks," Journal of Network and Systems Management, vol. 24, pp. 789-812, 2016.

[17] K. Xie, J. Cao, X. Wang, J. Wen. "Optimal Resource Allocation for Reliable and Energy Efficient Cooperative Communications." IEEE Transactions on Wireless Communications, vol. 12, no. 10, pp. 49945007, 2013.

[18] H.Y. Xin and X.X. Liu, "Energy-balanced transmission with accurate distances for strip-based wireless sensor networks," IEEE Access, vol. 5, pp. 16193-16204, 2017.

[19] X. Chen, M. Ma, A. Liu. "Dynamic Power Management and Adaptive Packet Size Selection for IoT in eHealthcare," Computers \& Electrical Engineering, vol. 65, pp. 357-375, 2018.

[20] J. Wang, A. Liu, T. Yan, Z. Zeng. "A Resource Allocation Model Based on Double-sided Combinational Auctions for Transparent Computing," Peer-to-Peer networking and Applications, DOI: 10.1007/s12083017-0556-6, 2017.

[21] Q. Liu, A. Liu. "On the hybrid using of unicast-broadcast in wireless sensor networks," Computers \& Electrical Engineering, DoI: http://dx.doi.org/10.1016/j.compeleceng. 2017.03.004, 2017.

[22] D. Z. Zeng, L. Gu, L. Lian, S. Guo, H. Yao and J.K. Hu, "On CostEfficient Sensor Placement for Contaminant Detection in Water Distribution Systems," IEEE Transactions on Industrial Informatics, vol. 12, no. 6, pp. 2177-2185, 2016.

[23] T. Wang, Y. Li, G.J. Wang, J.N. Cao, M. Z. A. Bhuiyan and W.J. Jia, "Sustainable and Efficient Data Collection from WSNs to Cloud," IEEE Transactions on Sustainable Computing, DOI: 10.1109/TSUSC.2017.2690301, 2017.

[24] K. Xie, X. Wang, J. Wen, J. Cao, "Cooperative Routing with Relay Assignment in Multi-radio Multi-hop Wireless Networks", IEEE/ACM Transactions on Networking, vol. 24, no. 2, pp. 859-872, 2016.

[25] J.S. Gui, L.H. Hui and N. X. Xiong, "A Game-Based Localized MultiObjective Topology Control Scheme in Heterogeneous Wireless Networks,” IEEE Access, vol. 5, pp. 2396-2416, 2017.

[26] [14] X. Liu, Y. Liu, H. Song, A. Liu. "Big Data Orchestration as a Service Networking." IEEE Communications Magazine, vol. 55, no. 9, pp. 94101, 2017.

[27] F. Ma, X. Liu, A. Liu, M. Zhao, C. Huang, T. Wang. "A Time and Location Correlation Incentive Scheme for Deeply Data Gathering in Crowdsourcing Networks." Wireless Communications and Mobile Computing, vol. 2018, 2018, 8052620, DoI: 10.1155/2018/8052620.

[28] J. Xu, X. Liu, M. Ma, A. Liu, T. Wang, C. Huang. "Intelligent Aggregation based on Content Routing Scheme for Cloud Computing." Symmetry, vol. 9, no. 10, 221, 2017; doi:10.3390/sym9100221

[29] M. Huang, A. Liu, T. Wang, C. Huang. "Green Data Gathering under Delay Differentiated Services Constraint for Internet of Things." Wireless Communications and Mobile Computing, vol. 2018, 2018, 9715428, DoI: DoI: 10.1155/2018/9715428.

[30] M. Wu, Y. Wu, X. Liu, M. Ma, A. Liu, M. Zhao. "Learning Based Synchronous Approach from Forwarding Nodes to Reduce the Delay for Industrial Internet of Things." EURASIP Journal on Wireless Communications and Networking, vol. 2018, 2018, 10. DOI: 10.1186/s13638-017-1015-z..

[31] A. Liu, Z. Chen, N. Xiong. "An Adaptive Virtual Relaying Set Scheme for Loss-and-Delay Sensitive WSNs." Information Sciences, vol. 424, pp. 118-136, 2018.

[32] T. Li, Y . Liu, L. Gao, A. Liu. "A Cooperative-based Model for SmartSensing Tasks in Fog Computing," IEEE Access, vol. 5, pp. 2129621311, 2017.

[33] J.S. Gui and J. Deng, "A topology control approach reducing construction cost for lossy wireless sensor networks," Wireless Pers. Commun. vol. 95, vol. 3, pp. 2173-2202, 2017. 
[34] Z. Wang, Q. Cao, H. Qi, H. Chen, Q. Wang. "Cost-Eective Barrier Coverage Formation in Heterogeneous Wireless Sensor Networks." Ad Hoc Networks, vol. 64, no. 9, pp. 65-79, 2017.

[35] Y. Xu, X. Chen, A. Liu, C. Hu. "A Latency and Coverage Optimized Data Collection Scheme for Smart Cities Based on Vehicular Ad-hoc Networks." Sensors, 2017, 17 (4), 888, doi:10.3390/s17040888

[36] X. Liu, G. Li, S. Zhang, A. Liu. "Big Program Code Dissemination Scheme for Emergency Softwaredefine Wireless Sensor Networks," Peer-to-Peer networking and applications, DOI: 10.1007/s120830170565-5, 2017.

[37] Z. Wang, H. Chen, Q. Cao, H. Qi, Z. Wang, Q. Wang. "Achieving Location Error Tolerant Barrier Coverage for Wireless Sensor Networks." Computer Networks, vol. 112, no. 1, pp. 314-328, 2017.

[38] Z. Chen, M. Ma, X. Liu, A. Liu, M. Zhao. "Reliability Improved Cooperative Communications over Wireless Sensor Networks," Symmetry, vol. 9, no. 10, 209, 2017. doi:10.3390/sym9100209..

[39] M. Zhou, M. Zhao, A. Liu, M. Ma, T. Wang, C. Huang. "Fast and Efficient Data Forwarding Scheme for Tracking Mobile Target in Sensor Networks," Symmetry, vol. 9, no. 11, 269, 2017.

[40] Y. Zhang, S. He, J. Chen . "Data gathering optimization by dynamic sensing and routing in rechargeable sensor networks." IEEE/ACM Transactions on Networking, vol. 24, no.3, pp. 1632-1646, 2016.

[41] J. Tang, A. Liu, M. Zhao, T. Wang. "An Aggregate Signature based Trust Routing for Data Gathering in Sensor Networks." Security and Communication Networks. Vol. 2018, 6328504,, DoI: $10.1155 / 2018 / 6328504$.

[42] Z. Wang, J. Liao, Q. Cao, H. Qi, Z. Wang. "Achieving k-barrier Coverage in Hybrid Directional Sensor Networks." IEEE Transactions on Mobile Computing, vol. 13, no. 7, pp. 1443-1455, 2014.

[43] L. Pu, X. Chen, J. Xu, X. Fu, "D2D Fogging: An Energy-efficient and Incentive-aware Task Offloading Framework via Network-assisted D2D Collaboration," IEEE Journal on Selected Areas in Communications , Vol. 34, No. 12, pp. 3887 - 3901, 2016.

[44] X. Hu, T.H.S. Chu, V.C.M. Leung, E. C.-H. Ngai, P. Kruchten, and H.C.B. Chan, "A Survey on Mobile Social Networks: Applications, Platforms, System Architectures, and Future Research Directions." IEEE Communications Surveys \& Tutorials, vol. 17, no. 3, pp. 15571581, 2015.

[45] X. Chen, L. Pu, L. Gao, W. Wu, and D. Wu, "Exploiting Massive D2D Collaboration for EnergyEfficient Mobile Edge Computing," IEEE Wireless Communications, Vol. 24, No. 4, pp. 64 - 71, Aug. 2017.

[46] H. Dai, X. Wu, L. Xu, et al. "Practical scheduling for stochastic event capture in energy harvesting sensor networks." International Journal of Sensor Networks. Vol. 18, no. (1/2), pp. 85-100, 2015

[47] X. Liu. "A Deployment Strategy for Multiple Types of Requirements in Wireless Sensor Networks." IEEE Transactions on Cybernetics, vol. 45, no. 10, pp. 2364-2376, 2015.

[48] M. Dong, X. Liu, Z. Qian, et al. "QoE ensured Price Competition Model for Emerging Mobile Networks." IEEE Wireless Communications, vol. 22, no. 4, pp. 50-57, 2015.

[49] S. He, X. Gong, J. Zhang, J. Chen. "Curve based Deployment for Barrier Coverage in Wireless Sensor Networks." IEEE Transactions on Wireless Communications, vol. 13, no. 2, pp. 724-735, 2014.

[50] Y. Liu, M. Dong, K. Ota, A. Liu. "ActiveTrust: Secure and Trustable Routing in Wireless Sensor Networks." IEEE Transactions on Information Forensics and Security, vol. 11, no. 9, pp. 2013-2027, 2016.

[51] X. Liu, M. Dong, K. Ota, et al. "Service Pricing Decision in CyberPhysical Systems: Insights from Game Theory." IEEE Transactions on Services Computing. Vol. 9, no. 2, pp. 186-198, 2016.

[52] M. Dong, K. Ota, A. Liu, M. Guo. "Joint Optimization of Lifetime and Transport Delay under Reliability Constraint Wireless Sensor Networks." IEEE Transactions on Parallel and Distributed Systems, vol. 27, no. 1, pp. 225-236, 2016.

[53] J. Xu, A. Liu, N. Xiong, T. Wang, Z. Zuo. "Integrated Collaborative Filtering Recommendation in Social Cyber-Physical Systems." International Journal of Distributed Sensor Networks, Vol. 13, no. 12 , 2017. http://journals.sagepub.com/doi/full/10.1177/1550147717749745. 
[54] S. Lai, B. Ravindran, H. Cho. "Heterogenous quorum-based wake-up scheduling in wireless sensor networks." IEEE Transactions on Computers, vol. 59, no. 11, pp. 1562-1575, 2010.

[55] C. Chao, Y. Lee . "A quorum-based energy-saving MAC protocol design for wireless sensor networks." IEEE Transactions on Vehicular Technology, vol. 59, no. 2, pp. 813-822, 2010.

[56] CH. Tsai, TW. Hsu, MS. Pan, et al. "Cross-layer, energy-efficient design for supporting continuous queries in wireless sensor networks: a quorum-based approach." Wireless personal communications, vol. 51, no. 3, pp. 411-426, 2009.

[57] JR. Jiang. "Expected quorum overlap sizes of quorum systems for asynchronous power-saving in mobile ad hoc networks." Computer Networks, vol. 52, no. 17, pp. 3296-3306, 2008.

[58] GH. Ekbatanifard, R. Monsefi, MH. Yaghmaee, et al. "Queen-MAC: A quorum-based energy-efficient medium access control protocol for wireless sensor networks." Computer Networks, vol. 56, no. 8, pp. 2221-2236, 2012.

[59] Swain, R. R., Mishra, S., Samal, T. K., Kabat, M. R. (2014). Adv-MMAC: An advertisement based multichannel MAC protocol for wireless sensor networks. In IEEE, Contemporary computing and informatics (IC3I), 2014 international conference (pp. 347-352).

[60] Ray, S., Demirkol, I., \& Heinzelman, W. (2011). ADV-MAC: Analysis and optimization of energy efficiency through data advertisements for wireless sensor networks. Ad Hoc Networks, 9(5), 876-892.

[61] Ekbatanifard, G. H., Monsefi, R., Yaghmaee, M. H., \& Hosseini, S. A. (2012). Queen-MAC: A quorumbased energy-efficient medium access control protocol for wireless sensor networks. Computer Networks, 56(8), 2221-2236.

[62] Ray, S., Demirkol, I., \& Heinzelman, W. (2013). Supporting bursty traffic in wireless sensor networks through a distributed advertisement-based TDMA protocol (ATMA). Ad Hoc Networks, 11(3), 959-974.

\section{Authors' Profiles}

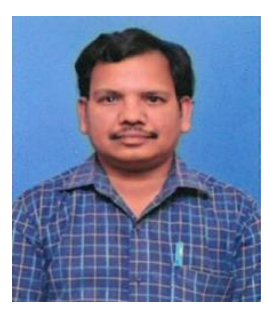

Ch.Rambabu, is a Research Scholar, pursuing Ph. D in the field of Wireless Sensor Networks from Jawaharlal Nehru Technological University, Kakinada, Andhra Pradesh, India. He has been working as an Academician in the capacity of a Senior Grade Assistant Professor in Electronics and Communication department of Gudlavalleru Engineering College, a reputed Autonomous Engineering College in Andhra Pradesh, India. He received B.Tech. (ECE) degree from Nagarjuna University, Guntur and M.Tech.(Digital Electronics and Communication Systems) degree from JNT University, Hyderabad. His keen interests are inclined more towards Wireless Communications and Signal Processing.

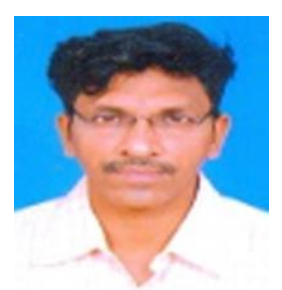

Dr. V.V.K.D.V. Prasad, working as a Professor and Head of the Department of Electronics \& Communication Engineering, in Gudlavalleru Engineering College, an Autonomous NBA accredited College in Andhra Pradesh, India. He received Ph. D for his work in Signal Processing in 2011 from Jawaharlal Nehru technological University, Kakinada, India. His areas of Interest include Signal Processing, Electrostatics, Electromagnetic fields and Transmission lines. He developed an abstract technical trait that addresses various other fields where digitalization can be achieved. His research findings are in the methodology used, problems encountered and the practical implications of composite features and filtering coefficients in advanced filters. 


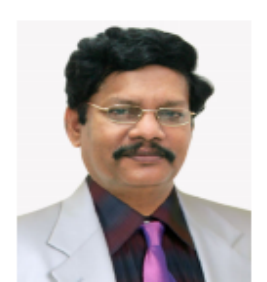

Dr. K. Satya Prasad received B.Tech. (ECE) degree from JNT University, Hyderabad, Andhra Pradesh, India in 1977, M.E. (Communication systems) from University of Madras, India in 1979, Ph.D. from IIT-Madras, India in 1989. He has more than 35 years of experience in teaching and 20 years in R\&D. His current research interests include Signals \& Systems, Communications, Digital signal processing, RADAR and Telemetry. He worked as Professor of Electronics \& Communication Engineering \& Former RECTOR, JNTUK and former Pro-Vice Chancellor, KLEF. At present he is professor of ECE and Rector, Vignan's Foundation for Science, Technology and Research (Deemed to be University), Vadlamudi, Andhra Pradesh.

How to cite this paper: Ch Rambabu, V.V.K.D.V.Prasad, K.Satya Prasad, " Multipath Cluster-based Hybrid MAC Protocol for Wireless Sensor Networks ", International Journal of Wireless and Microwave Technologies(IJWMT), Vol.10, No.1, pp. 1-16, 2020.DOI: 10.5815/ijwmt.2020.01.01 